\title{
The Keck Array: a pulse tube cooled CMB polarimeter
}

\section{Citation}

Sheehy, C. D., Peter AR Ade, R. W. Aikin, M. Amiri, S. Benton, C. Bischoff, J. J. Bock et al. 2010. "The Keck Array: a pulse tube cooled CMB polarimeter." In Proceedings of SPIE Millimeter, Submillimeter, and Far-Infrared Detectors and Instrumentation for Astronomy V, San Diego, California, June 27, 2010: 77411R.

\section{Published Version}

doi:10.1117/12.857871

\section{Permanent link}

http://nrs.harvard.edu/urn-3:HUL.InstRepos:33717521

\section{Terms of Use}

This article was downloaded from Harvard University's DASH repository, and is made available under the terms and conditions applicable to Other Posted Material, as set forth at http:// nrs.harvard.edu/urn-3:HUL.InstRepos:dash.current.terms-of-use\#LAA

\section{Share Your Story}

The Harvard community has made this article openly available.

Please share how this access benefits you. Submit a story.

\section{Accessibility}




\title{
The Keck Array: a pulse tube cooled CMB polarimeter
}

C. D. Sheehy, ${ }^{a, b}$ P. A. R. Ade ${ }^{c}$ R. W. Aikin, ${ }^{d}$ M. Amiri, ${ }^{e}$ S. Benton, ${ }^{f}$ C. Bischoff,${ }^{g}$

J. J. Bock ${ }^{d}, h$ J. A. Bonetti ${ }^{h}$ J. A. Brevik,${ }^{d}$ B. Burger,${ }^{e}$ C. D. Dowell $,{ }^{d}, h$ L. Duband ${ }^{i}$ J. P. Filippini, ${ }^{d}$ S. R. Golwala,${ }^{d}$ M. Halpern,${ }^{e}$ M. Hasselfield,${ }^{e}$ G. Hilton,${ }^{j}$ V. V. Hristov ${ }^{d}$ K. Irwin, ${ }^{j}$ J. P. Kaufman ${ }^{k}$ B. G. Keating, ${ }^{k}$ J. M. Kovac,${ }^{g}$ C. L. Kuo $,{ }^{l}, m$ A. E. Lange,${ }^{d}$ E. M. Leitch ${ }^{a}$ M. Lueker, ${ }^{d, h}$ C. B. Netterfield, ${ }^{f}$ H. T. Nguyen,${ }^{d, h}$ R. W. Ogburn IV,${ }^{l, m}$ A. Orlando ${ }^{d}$ C. L. Pryke,${ }^{b}$ C. Reintsema,${ }^{j}$ S. Richter ${ }^{g}$ J. E. Ruhl,${ }^{n}$ M. C. Runyan,${ }^{d}$ Z. Staniszewski, ${ }^{d, h}$ S. Stokes, ${ }^{l, m}$ R. Sudiwala,${ }^{c}$ G. Teply, ${ }^{d}$ K. L. Thompson, ${ }^{l, m}$ J. E. Tolan,,${ }^{l, m}$ A. D. Turner, ${ }^{h}$ P. Wilson, ${ }^{h}$ and C. L. Wong ${ }^{g}$

${ }^{a}$ University of Chicago, KICP, 933 E. 56th St., Chicago, IL 60637 USA;

${ }^{b}$ School of Physics \& Astronomy, University of Minnesota, 116 Church Street S.E., Minneapolis, MN 55455;

${ }^{c}$ Dept. of Physics and Astronomy, University of Wales, Cardiff, CF24 3YB, Wales, UK; ${ }^{d}$ California Institute of Technology, 1200 E. California Blvd., Pasadena, CA 91125 USA;

${ }^{e}$ Department of Physics \& Astronomy, University of British Columbia, 6224 Agricultural Road, Vancouver, BC V6T1Z1, Canada;

${ }^{f}$ Department of Physics, University of Toronto, Toronto, ON M5S 1A7, Canada;

${ }^{g}$ Harvard-Smithsonian Center for Astrophysics, 60 Garden Street, Cambridge, MA 02138;

${ }^{h}$ Jet Propulsion Laboratory, 4800 Oak Grove Dr., Pasadena, CA 91109, USA;

${ }^{i}$ Service des Basses Tempratures, DRFMC, CEA-Grenoble, 17 rue des Martyrs, 38054 Grenoble Cedex 9, France;

${ }^{j}$ NIST Quantum Devices Group, 325 Broadway, Boulder, CO 80305, USA;

${ }^{k}$ University of California, San Diego, La Jolla, CA 92093, USA;

'Stanford University, Stanford, 382 Via Pueblo Mall, CA 94305, USA;

${ }^{m}$ Kavli Institute for Particle Astrophysics and Cosmology (KIPAC), Sand Hill Road 2575, Menlo Park, CA 94025, USA;

${ }^{n}$ Physics Department, Case Western Reserve University, Cleveland, OH 44106 USA

\begin{abstract}
The Keck Array is a cosmic microwave background (CMB) polarimeter that will begin observing from the South Pole in late 2010. The initial deployment will consist of three telescopes similar to BICEP2 housed in ultracompact, pulse tube cooled cryostats. Two more receivers will be added the following year. In these proceedings we report on the design and performance of the Keck cryostat. We also report some initial results on the performance of antenna-coupled TES detectors operating in the presence of a pulse tube. We find that the performance of the detectors is not seriously impacted by the replacement of BICEP2's liquid helium cryostat with a pulse tube cooled cryostat.
\end{abstract}

\section{INTRODUCTION}

The presence of a curl component in the polarization pattern of the CMB is a generic prediction of inflation. ${ }^{1,2}$ A detection of this so-called B-mode of the CMB polarization would not only provide "smoking gun" style evidence that inflation occurred, but also offer an important probe of GUT scale physics inaccessible to laboratory experiments. The Keck Array is optimized to detect the degree scale peak of the inflationary B-mode spectrum,

Send correspondence to C. D. Sheehy,

E-mail: csheehy@uchicago.edu

Millimeter, Submillimeter, and Far-Infrared Detectors and Instrumentation for Astronomy V, edited by Wayne S. Holland, Jonas Zmuidzinas, Proc. of SPIE Vol. 7741, 77411R (C) 2010 SPIE · CCC code: 0277-786X/10/\$18 - doi: 10.1117/12.857871

Proc. of SPIE Vol. 7741 77411R-1 

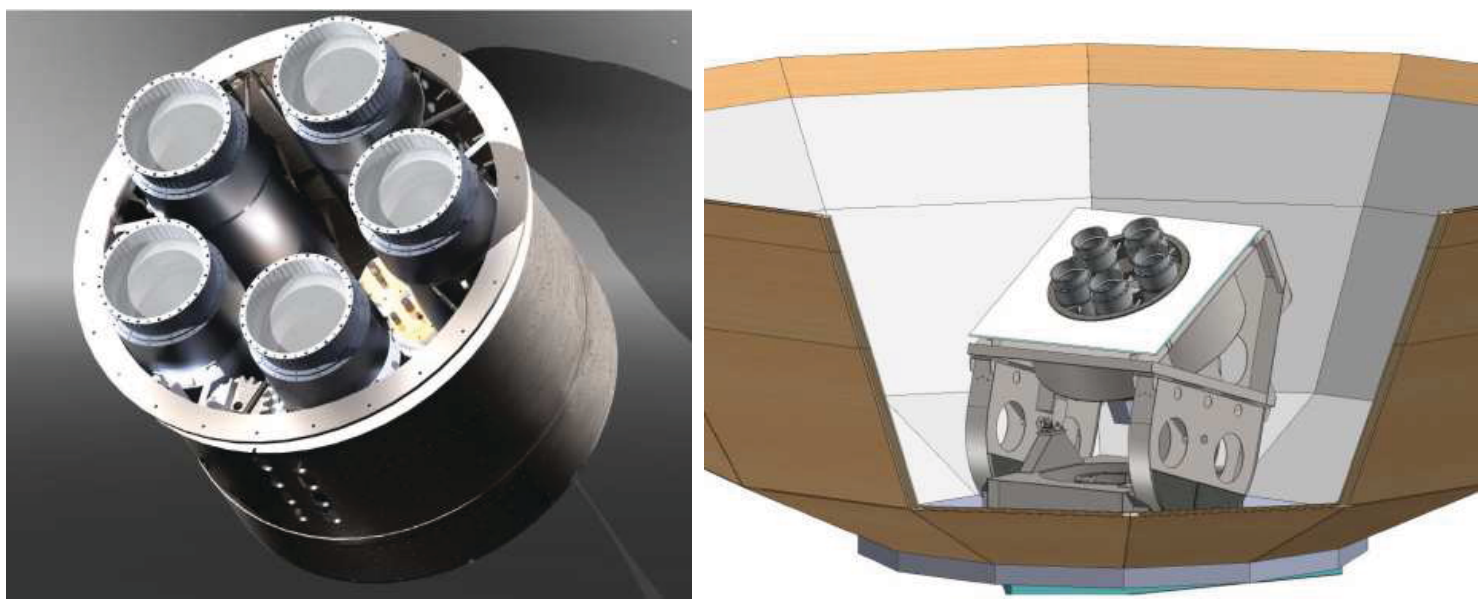

Figure 1. Left: CAD rendering of the modified drum of the DASI mount with 5 Keck cryostats installed. Right: Keck cryostats installed in the DASI mount and ground shield (cutaway).

the amplitude of which is parameterized by the ratio of tensor to scalar perturbations in the early universe, $r \sim T / S$. The simplest models of single field, slow roll inflation favor values of $r \leq 0.1$. No B-mode polarization has yet been detected. The current best upper limit on the B-mode spectrum of $r<0.24$ has been set by WMAP7 from temperature anisotropy alone. ${ }^{3}$ To constrain $r$ further will require sensitive measurements of the CMB polarization.

BICEP2, which deployed to the South Pole in November 2009 and will observe for the next two years, was designed with the explicit goal of detecting inflationary B-modes. ${ }^{4}$ Its optical design follows from BICEP1, which has constrained B-modes to $r<0.72 .{ }^{5}$ BICEP2's small aperture $(25 \mathrm{~cm})$ and on-axis, cold, refracting optics are ideal for characterizing and minimizing instrument systematics. The optics, which consist of anti-reflection coated lenses and infrared blocking filters, are optimized for a single frequency. ${ }^{6}$ The focal plane consists of 512 antenna-coupled, TES bolometers operating at $150 \mathrm{GHz}$, and are described in detail in Ref. 7. Each detector is sensitive to a single polarization orientation. Differencing the signal between members of 256 pairs of spatially coincident detectors allows for a high level of rejection of common mode noise. The initial performance of the BICEP2 detectors is described in Ref. 8.

Detecting the B-mode polarization of the CMB will require unprecedented sensitivity. To achieve this, the Keck Array will deploy five BICEP2 style receivers, beginning with three in November 2010 and following with two more in late 2011. These receivers will be fit into an existing mount at the South Pole originally built for the DASI experiment and most recently used for the QUAD experiment. ${ }^{9,10}$ The azimuth/elevation mount has a drum which can rotate about its axis, thus duplicating BICEP1 and BICEP2's ability to rotate the entire optics around the boresight. The mount with five receivers installed is shown in Figure 1. Because of the space constraints imposed by the existing infrastructure, maximizing the number of receivers requires designing a very compact cryostat. For this reason, and because the South Pole station does not have the resources to provide enough liquid helium to keep many receivers cold through the 9 months during which the station is inaccessible, the Keck Array will use pulse tube cooled cryostats in place of the liquid cryogen cryostats of BICEP1 and BICEP2.

Section 2 discusses the design of the Keck cryostat and its thermal performance. Section 3 discusses the initial performance of BICEP2 detectors operating in the cryostat, paying specific attention to any possible degradation in performance compared to BICEP2.

\section{CRYOSTAT DESIGN}

The Keck cryostat, shown in cross section in Figure 2, uses a Cryomech PT410 two-stage pulse tube cooler, mounted on vibration isolating bellows. According to Cryomech's published cooling capacity curves, the first 


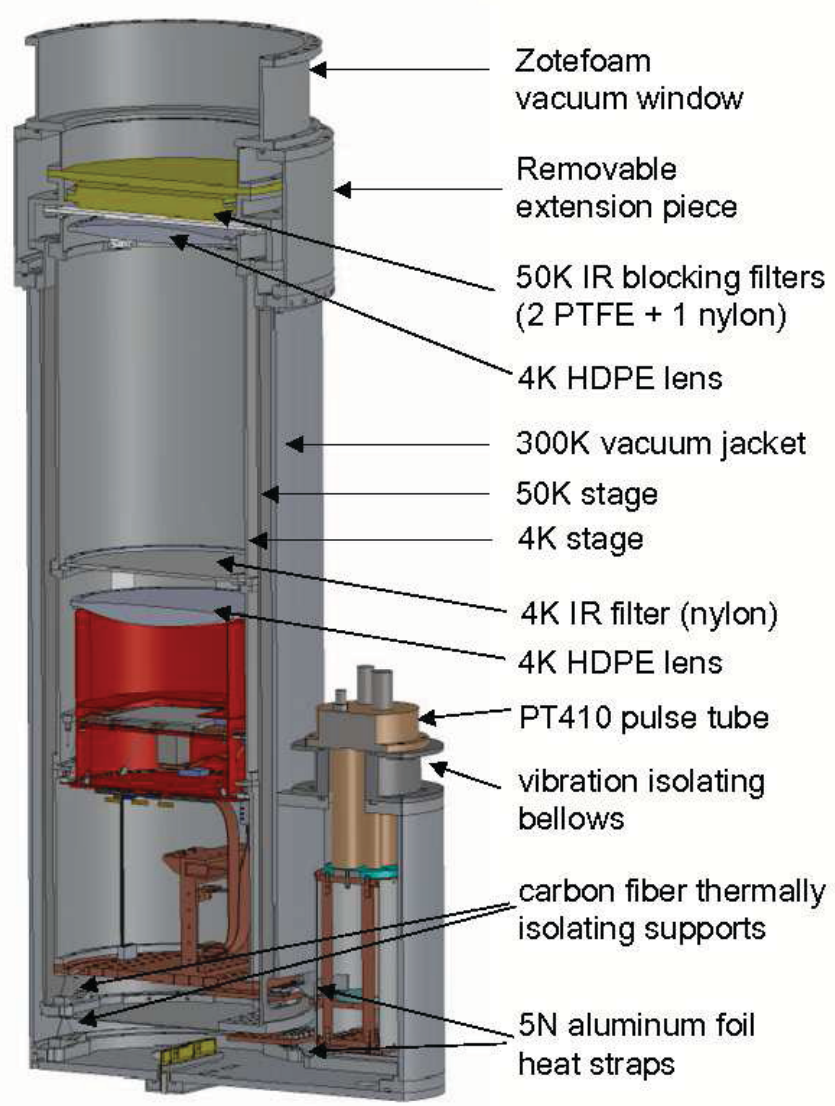

Figure 2. Cross section of the Keck cryostat.

stage of the PT410 will maintain a temperature $T<50 \mathrm{~K}$ while conducting as much as $45 \mathrm{~W}$ of power. This substantial amount of cooling power allows us to thermally sink three separate infrared blocking filters to the $50 \mathrm{~K}$ stage of the pulse tube. In BICEP2 these filters are mounted to liquid helium vapor cooled stages. Also according to the system specifications given by Cryomech, the 2nd stage of the PT410 maintains a temperature of $\sim 3.5 \mathrm{~K}$ at $0.5 \mathrm{~W}$ of conducted power, rising to $\sim 4.25 \mathrm{~K}$ at $1.0 \mathrm{~W}$ of power, nearly independent of the power on the 1st stage. We have verified this in the lab for a nominal loading of $25 \mathrm{~W}$ on the 1st pulse tube stage. Because of the finite cooling capacity of the PT410 we have taken great care in the design of our mechanical supports to minimize parasitic loads onto the $4 \mathrm{~K}$ stage, and in the design of our heat straps to maximize their thermal conductivity.

A removable cryostat extension encloses the filter stack and the top of the optics tube. This extension could be swapped out for one of a different height, providing flexibility in filter design and allowing the possible addition of a stepped half wave plate.

The lack of a tank for liquid cryogens has allowed us to highly compactify the cryostat. The largest diameter of the BICEP2/Keck insert is 14.76" and the main body of the Keck cryostat has an outer diameter of 18.0", leaving very little clearance between stages. The largest diameter of the cryostat, not including the off-axis "doghouse" housing the pulse tube, is the 19.5" diameter of the extension piece and its mating flange.

Magnetic shielding inside the cryostat is a sheet of rolled Amuneal A4K, which we have clamped to the $50 \mathrm{~K}$ cylinder wall, drilled in place (mostly through pre-drilled holes in the shielding), and attached with low profile screws. The two ends of the A4K shield overlap by a few inches. The geometry of this shield is different from BICEP2, which is a rolled and welded cylinder. Also, because Amuneal has phased out Cryoperm and replaced 

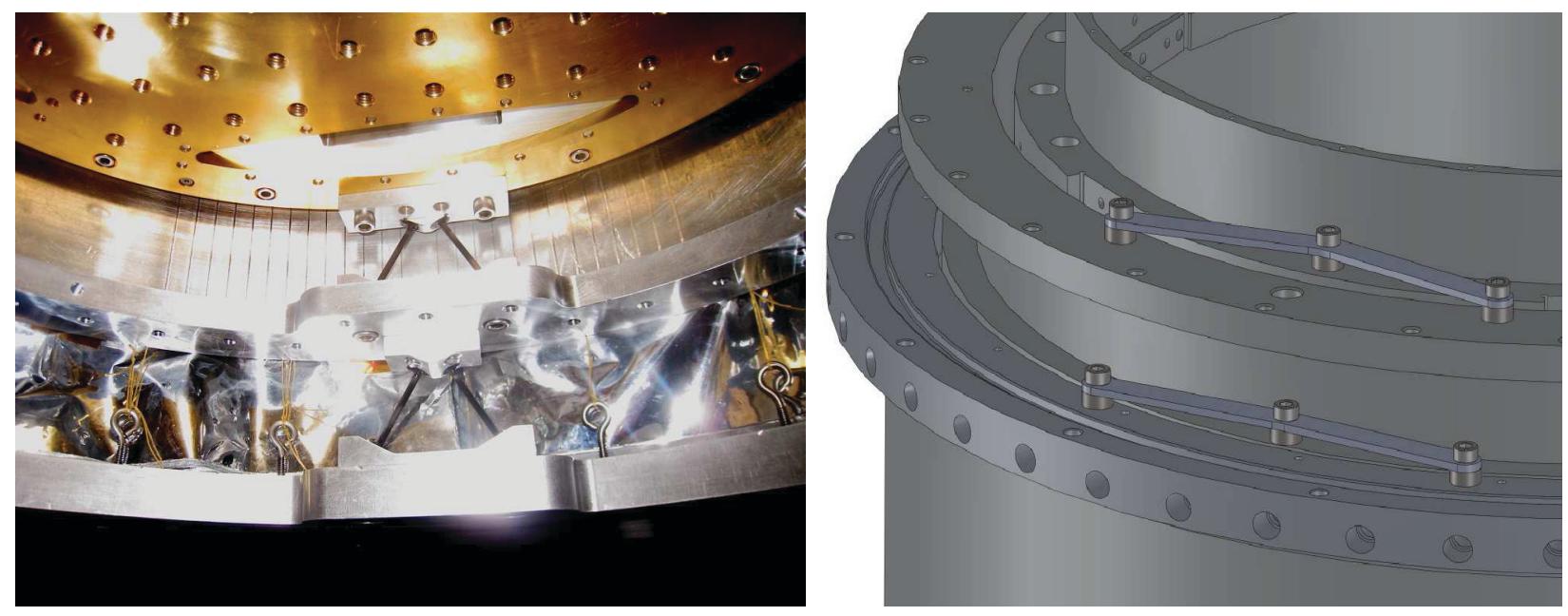

Figure 3. Left: Carbon fiber mechanical supports at the back end of the cryostat. The bottom standoff is one of three two-member trusses thermally isolating the $50 \mathrm{~K}$ assembly from the $300 \mathrm{~K}$ vacuum jacket. The top standoff is one of the three trusses supporting the $4 \mathrm{~K}$ assembly off of the $50 \mathrm{~K}$ assembly. Right: Titanium 6-Al-4V mechanical supports at the top of the cryostat. These constrain the top of the cold stages in the radial direction while allowing a large amount of differential thermal contraction in the axial direction.

it with $\mathrm{A} 4 \mathrm{~K}$, which is supposedly both higher performance and cheaper, the use of A4K in the Keck cryostat is a difference from BICEP2.

\subsection{THERMALLY ISOLATING MECHANICAL SUPPORTS}

Each cold stage is mechanically supported at the back end of the cryostat by three sets of two-member carbon fiber trusses. These trusses are shown in the left hand panel of Figure 3. Each truss consists of two .108" diameter by 2.084 " length carbon fiber rods epoxied into .141" diameter by .310" deep holes drilled into aluminum blocks.

The properties of carbon fiber at temperatures of $4 \mathrm{~K}$ and below were investigated in detail by Ref. 11 . The ratio of elastic modulus to thermal conductivity of carbon fiber is very high at cryogenic temperatures. At $4 \mathrm{~K}$ this ratio is higher than any other other material tested in Ref. 11, including G10 and Vespel. At $50 \mathrm{~K}$ this ratio drops but is still comparable to G10. The successful use of carbon fiber as thermally isolating truss supports for the sub-Kelvin stages of the SPIDER and BICEP2 focal planes motivated its use in the Keck cryostats. FEA analysis of the carbon fiber trusses indicates they will provide sub-arc-minute pointing accuracy and a mechanical strength adequate for a $300 \mathrm{lb}$ load with a safety factor greater than five. We have not measured their thermal conductivity, but a simple calculation predicts of order $16 \mathrm{~mW}$ conducted from the $50 \mathrm{~K}$ to $4 \mathrm{~K}$ assembly.

The cold stages of the cryostat are supported at the front end by three v-shaped titanium 6-Al-4V "boomerangs," shown in the right hand panel of Figure 3. These pieces are very stiff in the radial direction but quite flexible along the cryostat's axis, an acceptable configuration because of the stiffness of the rear carbon fiber supports in the axial direction. The flexibility of the titanium supports in the axial direction safely allows a large ( $0.2 ")$ differential thermal contraction between the $300 \mathrm{~K}$ and $50 \mathrm{~K}$ stages. Titanium was chosen for its high stiffness and for its large yield-strength to thermal conductivity ratio at low temperatures, which was needed to accommodate the radial differential thermal contraction between stages. The total loading onto $4 \mathrm{~K}$ from the titanium supports is estimated from a simple calculation to be $35 \mathrm{~mW}$.

\subsection{ALUMINUM FOIL HEAT STRAPS}

The liquid helium tank in the BICEP2 cryostat provides a large surface on which to directly mount the $4 \mathrm{~K}$ receiver. The Keck cryostat has no such surface. As such we have designed a very high thermal conductivity heat strap to connect the pulse tube cold head to the BICEP2 receiver. Running contrary to this goal is the need for the heat strap to be flexible in order to avoid transmitting vibrations from the pulse tube to the focal 


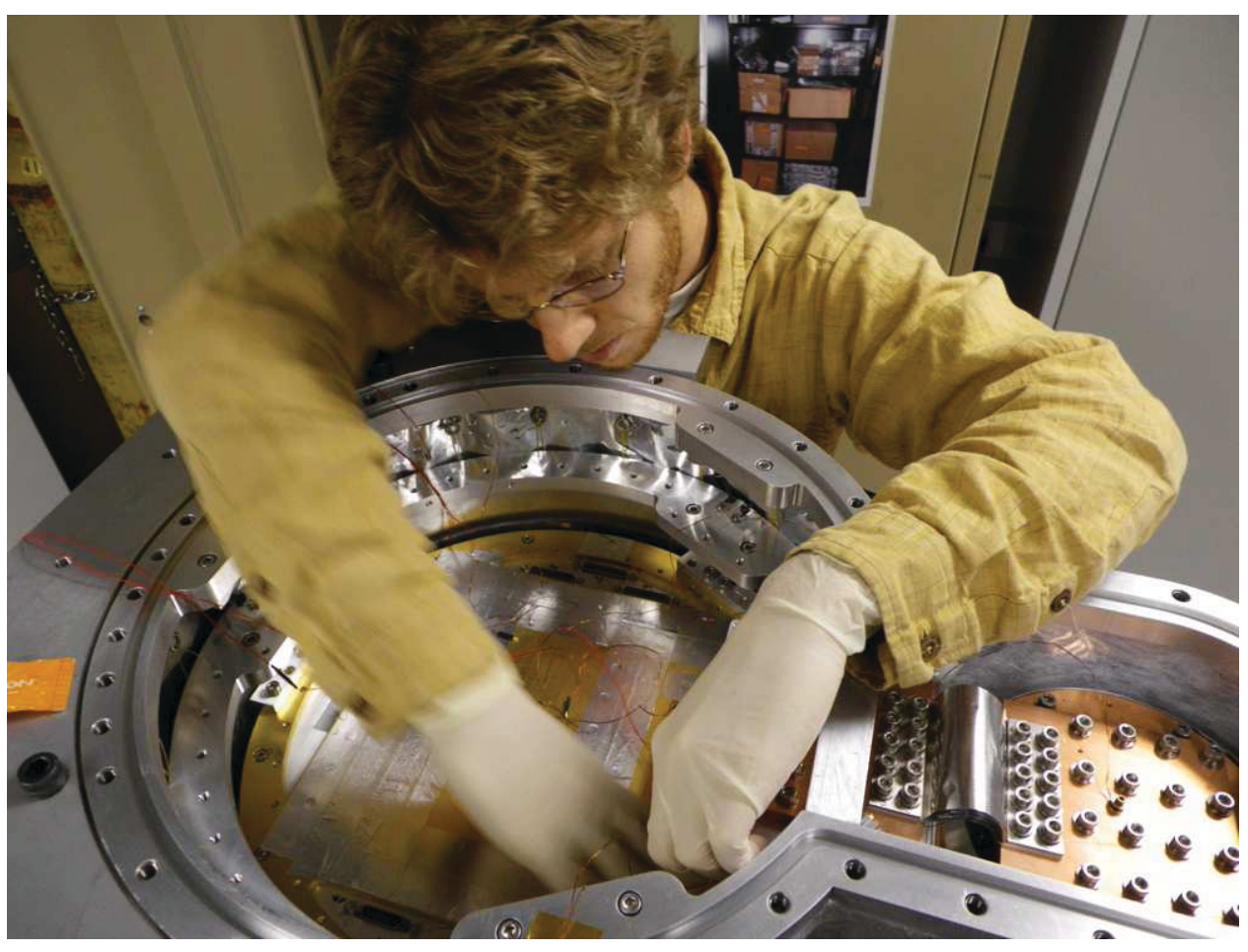

Figure 4 . The back of a Keck cryostat with the vacuum close-out plate removed, showing the $300 \mathrm{~K}$, $50 \mathrm{~K}$, and $4 \mathrm{~K}$ stages. The $50 \mathrm{~K}$ aluminum foil heatstrap is visible in the lower right corner.

plane. To satisfy both these competing criteria we have constructed our heat straps from stacks of $99.999 \%$ pure $(5 \mathrm{~N})$ aluminum foil. The stacks of foil are pressed against OFHC copper blocks at either end via an aluminum clamping plate and 10 18-8 stainless steel, 1/4"'-20 bolts tightened to a torque of $\sim 10 \mathrm{ft}-\mathrm{lbs}$. (We exceed the torque limit for the bolts because we are using nuts and not threaded holes.) The $50 \mathrm{~K}$ heat strap is shown in Figure 4. The strap is very flexible along the axis connecting the pulse tube cold head and the $4 \mathrm{~K}$ mating surface, as well as along the axial direction of the cryostat, and will transmit motion in these directions very weakly.

Great care is needed in the assembly of the heat straps to ensure good thermal contact between foils. To this end we have constructed an assembly jig that holds the foils against the copper blocks via the aluminum pressure plate during drilling. The pre-clamping of the foils prevents any edges from being raised. The pressure plate is then fastened in place with screws and nuts, and the heat strap can be removed from the jig.

The use of $5 \mathrm{~N} \mathrm{Al}$ was motivated by its very high thermal conductivity at $4 \mathrm{~K}$, which is approximately a factor 10 higher than OFHC copper with a residual resistivity ratio of $\mathrm{RRR}=50,{ }^{12,13}$ and by its low price and availability relative to $5 \mathrm{~N} \mathrm{Cu}$. A very low cross sectional area is needed to achieve an acceptable temperature rise, thus allowing us to maximize the flexibility of the strap. However, the use of high purity aluminum in cryogenic applications seems to be discouraged in the literature. ${ }^{12}$ The reason cited is that the oxide layer on aluminum gives it a very high thermal contact resistance, rendering it unsuitable for use unless extraordinary measures are taken. Although we have taken care to maximize the thermal contact between foils, the foils are not welded. Because the foils are stacked they mate to their end blocks in parallel. Thus the conductance of the straps is limited by the contact resistance between foils. Nonetheless, we have not observed unacceptably high contact resistances. A $4 \mathrm{~K}$ strap consisting of 10 foils, each 3.00" wide by $\sim 2.5$ " free length by .004" thick, was measured to have a conductance of $1.1 \mathrm{~W} / \mathrm{K}$ at $4 \mathrm{~K}$, with a temperature rise of $0.48 \mathrm{~K}$ across it while conducting $0.53 \mathrm{~W}$ of power. Given the range of thermal conductivities among different samples of $5 \mathrm{~N} \mathrm{Al}$, which is given by Ref. 12 to be $4-20 \times 10^{3} \mathrm{~W} / \mathrm{m} / \mathrm{K}$, our calculations imply that the contact conductance between foils at $4 \mathrm{~K}$ must be somewhere in the range $3-9 \mathrm{~W} / \mathrm{K}$. We have since added an identical 10 foil stack to the reverse 


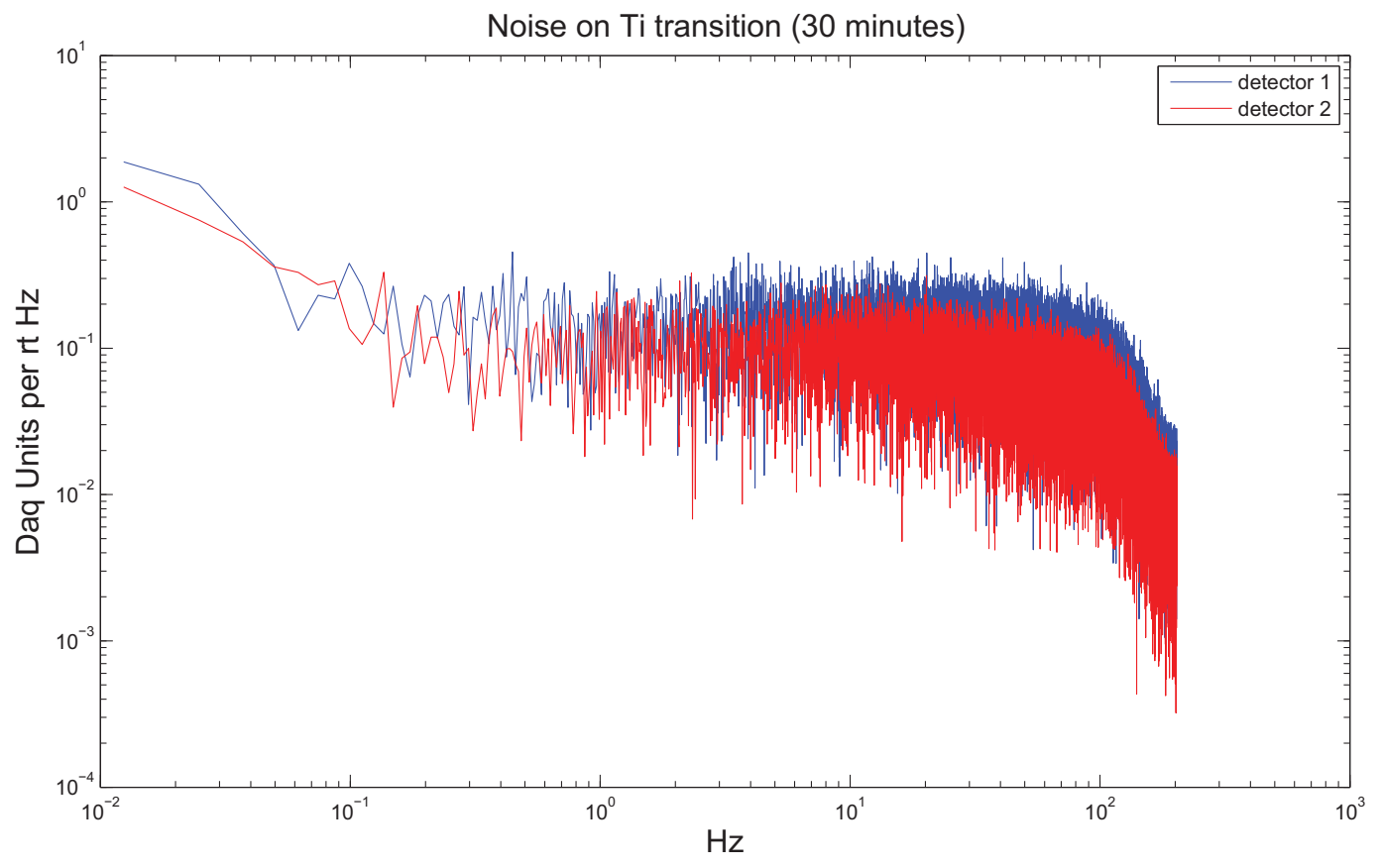

Figure 5. Noise PSDs of two detector pixels, which were shaded to allow them to be biased onto transition in the radiation environment of the lab. In neither curve is the $1.4 \mathrm{~Hz}$ pulse tube frequency visible.

sides of the strap to increase its conductance by a factor of two. The thermal contact resistance of high purity aluminum does not appear to be an insurmountable barrier to its use in high conductivity, flexible heat straps. To increase conductance further it would be possible to construct the end blocks from high purity aluminum (if not $5 \mathrm{~N} \mathrm{Al}$ then perhaps $\mathrm{Al} 1100)$ and e-beam weld the foils to the blocks.

\section{PERFORMANCE}

The major difference between BICEP2 and Keck is the use of a pulse tube cooled cryostat in place of a liquid helium cooled cryostat. We have performed initial commissioning of BICEP2 style detectors in the Keck cryostat. We have not yet observed any large differences in performance of the detectors while running them in the presence of a pulse tube as compared to BICEP2.

\subsection{DETECTOR NOISE}

One of the primary concerns regarding the performance of Keck as compared to BICEP2 is the possibility of elevated detector noise at the $1.4 \mathrm{~Hz}$ pulse tube frequency. We have measured the noise power spectral density (PSD) of two detector pixels, which are shown in Figure 5. A zotefoam vacuum window was in place on the cryostat and both pixels were shaded to allow the titanium TESs to be biased onto transition in the elevated loading conditions of the lab. ${ }^{14}$ The sampling rate was $406 \mathrm{~Hz}$. The PSDs shown are an average of 10 separate PSDs of $2^{16}$ samples each, for a total integration time of 27 minutes. In neither of the PSDs is the $1.4 \mathrm{~Hz}$ pulse tube frequency visible.

\subsection{MAGNETIC PICKUP}

There are differences between BICEP2 and Keck that might affect the response of the SQUID multiplexers to an external magnetic field. First, as discussed in Section 2, the magnetic shielding in the first Keck cryostat is of a slightly different geometry and size, and is made of a different material than that in BICEP2. Second, the pulse tube in the Keck cryostat introduces a large piece of stainless steel near the detectors. 

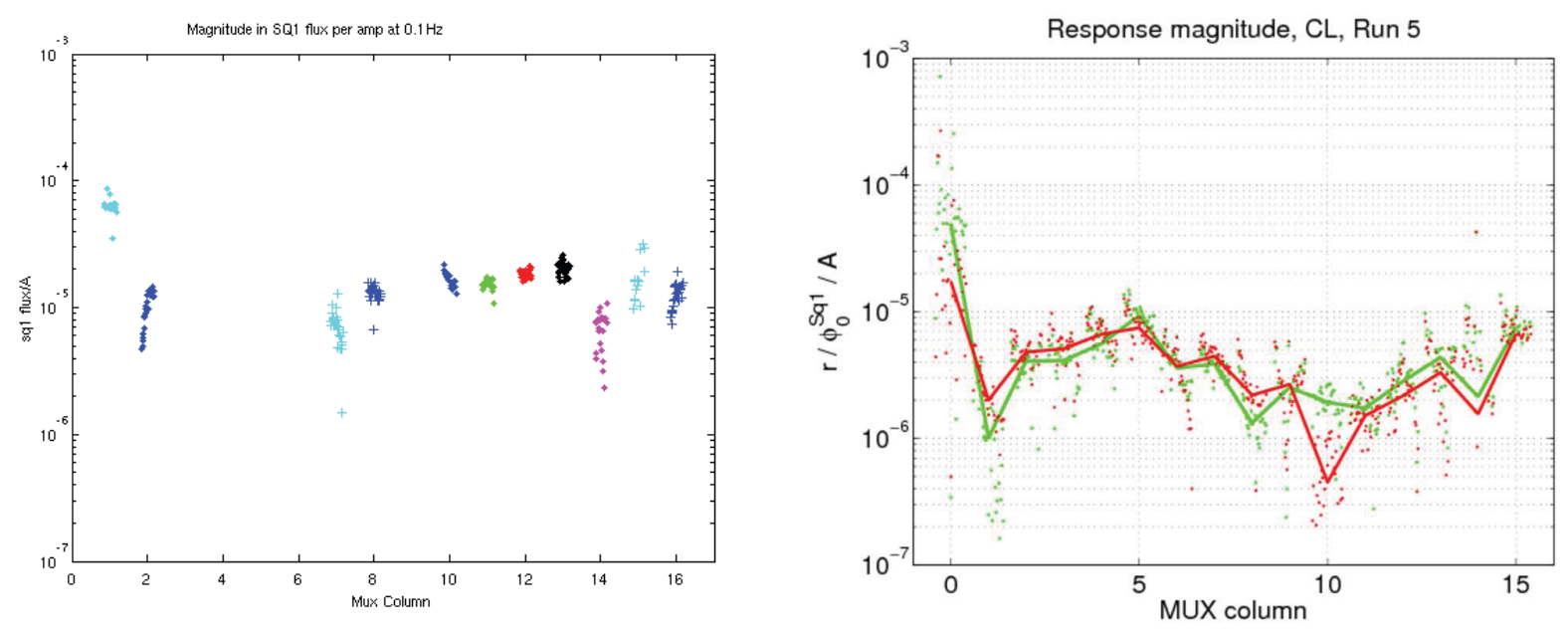

Figure 6. Left: SQUID response to a $0.1 \mathrm{~Hz}$ magnetic field in units of SQ1 $\phi_{0}$ per Amp peak-to-peak for the Keck cryostat, normalized to be comparable to a similar test performed for BICEP2. Right: The analogous test done for BICEP2.

We have tested the magnetic response of the SQUID multiplexers to a magnetic field induced by Helmholtz coils placed around the cryostat. The coils have $r=0.5 \mathrm{~m}$ and $N_{\text {turns }}=18$, operated with a current of 5.8 Amps peak-to-peak, and sweeping in frequency from 0.05 to $100 \mathrm{~Hz}$. Figure 6 shows the response of the SQ1 arrays operating closed-loop to the $0.1 \mathrm{~Hz}$ magnetic field, as well as the response from a similar test conducted for BICEP2. (Note that at the time this test was performed the Keck magnetic shielding was Cryoperm mounted to the $4 \mathrm{~K}$ assembly. This has subsequently been changed to A4K mounted at $50 \mathrm{~K}$. Also, the focal plane used in the BICEP2 run uses single turn series SQUID arrays, while the Keck run has three turn SSAs.) There is a factor $\sim 3$ increase in magnetic response of the Keck SQUIDs as compared to BICEP2. Another effect not shown here is that the Keck SQUID response does not appear to fall off with frequency as compared to BICEP2. However, we do not believe that either of these modest changes in SQUID response will significantly impact detector performance. Any residual magnetic response must be removed in analysis, which is discussed in detail in Ref. 4.

\section{CONCLUSION}

The Keck Array will deploy 3 BICEP2 style receivers to the South Pole in November 2010. The initial deployment will likely be three receivers at $150 \mathrm{GHz}$. Two more receivers will be added in November 2011. We have designed and tested ultra-compact, pulse tube cooled cryostats. The cryostat design allows the maximum possible number of receivers to be fit into the existing DASI mount at the South Pole station. Initial detector commissioning does not reveal any major impact on their performance arising from the new cryostats or the pulse tube, both of which constitute the main difference between Keck and BICEP2. Because of this, we expect the Keck Array to offer unprecedented sensitivity to the B-mode component of the CMB polarization.

\section{ACKNOWLEDGMENTS}

The Keck-Array is funded by the National Science Foundation through grants ANT-0742592 and ANT-0742818 and by the Keck Foundation. We acknowledge assistance from the KICP at the University of Chicago through the grant NSF PHY-0114422, and the support of the NASA Postdoctoral Program for Zak Staniszewski. This paper is dedicated to the memory of Andrew Lange, who died under tragic circumstances in January 2010. Andrew made invaluable contributions to the field of experimental cosmology. He played a central role in the conception of the BICEP2 and Keck experiments. His scientific aptitude, leadership, and unique abilities to recognize and develop young scientists are sorely missed by his friends and colleagues. 


\section{REFERENCES}

[1] Kamionkowski, M., Kosowsky, A., and Stebbins, A., "A Probe of Primordial Gravity Waves and Vorticity," Physical Review Letters 78, 2058-2061 (Mar. 1997).

[2] Seljak, U. and Zaldarriaga, M., "Signature of Gravity Waves in the Polarization of the Microwave Background," Physical Review Letters 78, 2054-2057 (Mar. 1997).

[3] Komatsu, E., Smith, K. M., Dunkley, J., Bennett, C. L., Gold, B., Hinshaw, G., Jarosik, N., Larson, D., Nolta, M. R., Page, L., Spergel, D. N., Halpern, M., Hill, R. S., Kogut, A., Limon, M., Meyer, S. S., Odegard, N., Tucker, G. S., Weiland, J. L., Wollack, E., and Wright, E. L., "Seven-Year Wilkinson Microwave Anisotropy Probe (WMAP) Observations: Cosmological Interpretation," ArXiv e-prints (Jan. 2010).

[4] Ogburn, R. W., et al., "The BICEP2 CMB polarization experiment," Proc. SPIE (2010).

[5] Chiang, H. C., Ade, P. A. R., Barkats, D., Battle, J. O., Bierman, E. M., Bock, J. J., Dowell, C. D., Duband, L., Hivon, E. F., Holzapfel, W. L., Hristov, V. V., Jones, W. C., Keating, B. G., Kovac, J. M., Kuo, C. L., Lange, A. E., Leitch, E. M., Mason, P. V., Matsumura, T., Nguyen, H. T., Ponthieu, N., Pryke, C., Richter, S., Rocha, G., Sheehy, C., Takahashi, Y. D., Tolan, J. E., and Yoon, K. W., "Measurement of Cosmic Microwave Background Polarization Power Spectra from Two Years of BICEP Data," "Astrophys. J" 711, 1123-1140 (Mar. 2010).

[6] Aikin, R. W., et al., "Optical performance of the BICEP2 telescope at the South Pole," Proc. SPIE (2010).

[7] Kuo, C. L., Bock, J. J., Bonetti, J. A., Brevik, J., Chattopadhyay, G., Day, P. K., Golwala, S., Kenyon, M., Lange, A. E., LeDuc, H. G., Nguyen, H., Ogburn, R. W., Orlando, A., Transgrud, A., Turner, A., Wang, G., and Zmuidzinas, J., "Antenna-coupled TES bolometer arrays for CMB polarimetry," Proc. SPIE $\mathbf{7 0 2 0}$ (Aug. 2008).

[8] Brevik, J. A., et al., "Initial performance of the BICEP2 antenna-coupled superconducting bolometers at the South Pole," Proc. SPIE (2010).

[9] Kovac, J. M., Leitch, E. M., Pryke, C., Carlstrom, J. E., Halverson, N. W., and Holzapfel, W. L., "Detection of polarization in the cosmic microwave background using DASI," Nature 420, 772-787 (Dec. 2002).

[10] Pryke, C., Ade, P., Bock, J., Bowden, M., Brown, M. L., Cahill, G., Castro, P. G., Church, S., Culverhouse, T., Friedman, R., Ganga, K., Gear, W. K., Gupta, S., Hinderks, J., Kovac, J., Lange, A. E., Leitch, E., Melhuish, S. J., Memari, Y., Murphy, J. A., Orlando, A., Schwarz, R., O’Sullivan, C., Piccirillo, L., Rajguru, N., Rusholme, B., Taylor, A. N., Thompson, K. L., Turner, A. H., Wu, E. Y. S., and Zemcov, M., "Second and Third Season QUaD Cosmic Microwave Background Temperature and Polarization Power Spectra," Astrophys. J 692, 1247-1270 (Feb. 2009).

[11] Runyan, M. C. and Jones, W. C., "Thermal conductivity of thermally-isolating polymeric and composite structural support materials between 0.3 and 4 K," Cryogenics 48, 448-454 (Sept. 2008).

[12] Woodcraft, A. L., "Recommended values for the thermal conductivity of aluminium of different purities in the cryogenic to room temperature range, and a comparison with copper," Cryogenics 45, 626-636 (Sept. 2005).

[13] Ekin, J. W., [Experimental Techniques for Low-Temperature Measurements], Oxford University Press, Oxford (2006).

[14] Orlando, A., et al., "Antenna-coupled TES bolometer arrays for BICEP2/Keck and Spider," Proc. SPIE (2010). 